Letter

\title{
Silicon nanocrystals as a photoluminescence down shifter for solar cells
}

\author{
Zhizhong Yuan a,*,1, Georg Pucker ${ }^{\mathrm{b}}$, Alessandro Marconi ${ }^{\mathrm{a}}$, Fabrizio Sgrignuoli ${ }^{\mathrm{a}}$, Aleksei Anopchenko ${ }^{\mathrm{a}}$, \\ Yoann Jestin ${ }^{\mathrm{b}}$, Lorenza Ferrario ${ }^{\mathrm{c}}$, Pierluigi Bellutti ${ }^{\mathrm{c}}$, Lorenzo Pavesi ${ }^{\mathrm{a}}$ \\ a Nanoscience Laboratory, Department of Physics, University of Trento, Via Sommarive 14, 38123 Povo, Trento, Italy \\ ${ }^{\mathrm{b}}$ Advanced Photonics and Photovoltaics Group, Bruno Kessler Foundation, Via Sommarive 18, 38123 Povo, Trento, Italy \\ ${ }^{\mathrm{c}}$ Microtechnologies Laboratory, Bruno Kessler Foundation, Via Sommarive 18, 38123 Povo, Trento, Italy
}

\section{A R T I C L E I N F O}

\section{Article history:}

Received 21 July 2010

Received in revised form

4 October 2010

Accepted 7 October 2010

Available online 6 January 2011

\section{Keywords:}

Photoluminescence

Solar cell

Silicon nanocrystal

Plasma-enhanced chemical vapor

deposition

Photoluminescence down shifter

\begin{abstract}
A B S T R A C T
The effects of a Si-rich silicon oxide (SRO) layer containing silicon nanocrystals as photoluminescence down-shifter layer on a conventional Si solar cell were investigated. Two SRO layers with different thicknesses but same composition were deposited on top of Si solar cells by plasma-enhanced chemical vapor deposition and followed by high temperature annealing to precipitate silicon nanocrystals. The SRO layers absorb efficiently high energy photons (especially higher than twice Si bandgap) and emit photons at longer wavelength, which are in turn absorbed by Si. A relative increase of about $14 \%$ to the internal quantum efficiency has been observed.
\end{abstract}

(c) 2010 Elsevier B.V. All rights reserved.

\section{Introduction}

Third generation solar cells are mainly based on bandgap engineering to improve conversion efficiency while still maintaining low cost $[1,2]$. There are many strategies to realize third generation devices [3]. One of the most promising is based on low dimensional nanostructures [4-8]. With the nanostructures one can address a problem that affects conventional Si solar cells, i.e. the inefficient use of high energy photons [9]. Indeed, silicon nanostructures are efficient photo-emitters in the visible region and have the potential to be used as photoluminescence $(\mathrm{PL})$ down shifter (PDS). With a PDS layer, high energy photons will be absorbed to emit low energy photons. These photons are then absorbed by the standard solar cell which results in an increased conversion efficiency. In Ref. [10] a 10\% increase in the short circuit current $\left(I_{\mathrm{SC}}\right)$ by quantum dot embedded plastic layer on top of a $\mathrm{Si}$ solar cell was modeled. Moreover, an improvement in the spectral response has been observed in solar cell covered by a spin-on-glass with silicon nanocrystals (Si-NCs) [11]. The conversion efficiency is increased by $0.4 \%$ with improved internal quantum efficiency (IQE) for photons with wavelengths shorter than $425 \mathrm{~nm}$. This promising result motivates the study in this letter about the down-shifting

\footnotetext{
* Corresponding author. Tel.:+39 461 282708; fax:+39 461282967.

E-mail address: zhizhong.yuan@gmail.com (Z. Yuan).

${ }^{1}$ Permanent address: Jiangsu University, Zhenjiang 212013, Jiangsu, PR China.
}

properties of plasma-enhanced chemical vapor deposition (PECVD) produced Si-NCs.

\section{Experimental procedure}

The cross-section of the PDS solar cell studied in this work is shown schematically in Fig. 1. Solar cells were obtained using floating zone (FZ) silicon substrates $(280 \mu \mathrm{m},\langle 100\rangle$, front-side polished, resistivity $0.3-0.7 \Omega \mathrm{cm}$, p-type), the back-side was boron implanted resulting in a sheet resistance of $10 \Omega / \square$ measured at the end of the processing. The top-emitter was realized by phosphor diffusion (sheet resistance $80 \Omega / \square$ ). The silicon rich oxide (SRO) layer was deposited by PECVD. The ratio of gas flows between $\mathrm{N}_{2} \mathrm{O}$ and $\mathrm{SiH}_{4}$ during the deposition process is 15 . The activation of boron and phosphor together with the growth of $\mathrm{Si}$ NCs was performed by thermal annealing at $900{ }^{\circ} \mathrm{C}$ for 30 min in $\mathrm{N}_{2}$ atmosphere. The SRO on top of the emitter was etched by reactive ion etching before metallization (Al deposited by DC-sputtering). The front metal is composed of metal fingers with two larger bus bars for current collection. The back-side of the cell is completely covered by Al. The size of the cells is $4.25 \mathrm{~mm} \times 4.25 \mathrm{~mm}$, with an active area of $3.70 \mathrm{~mm} \times 4.25 \mathrm{~mm}$.

Three devices were studied: (1) a cell with $105 \mathrm{~nm}$ thick $\mathrm{SiO}_{2}$ layer, which plays the role of the reference cell (labeled REF), (2) a cell with a thin (235 nm) SRO layer (labeled PDS-1), and (3) a device with a thick (677 $\mathrm{nm}$ ) SRO layer (labeled PDS-2). 


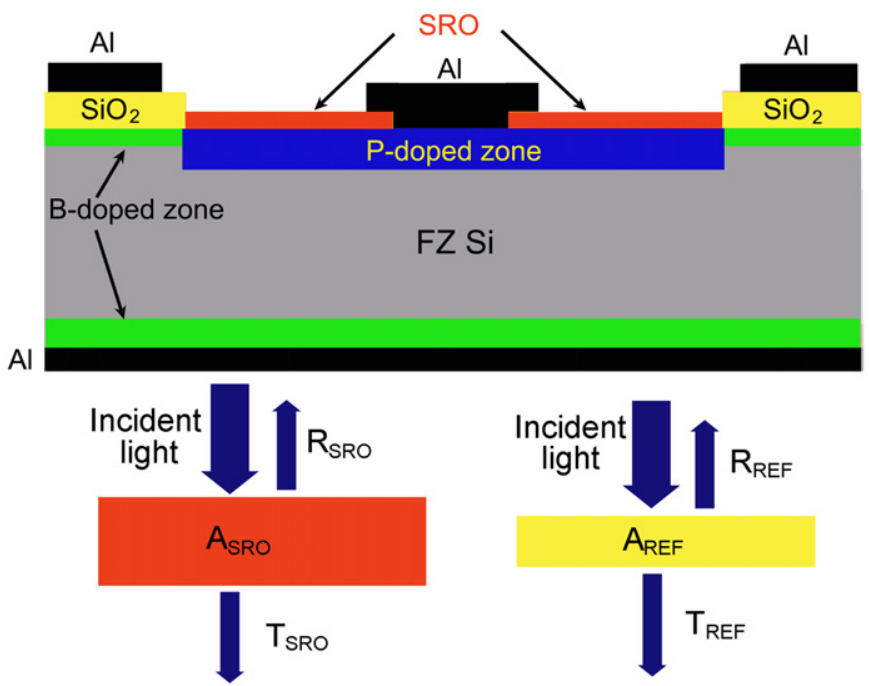

Fig. 1. (Top panel) schematic cross-section of the solar cell under study when covered by a silicon rich silicon oxide (SRO) layer (red). (Bottom panel) the different optical functions for the SRO layer (left) or the $\mathrm{SiO}_{2}$ layer (right). (For interpretation of the references to color in this figure legend, the reader is referred to the web version of this article.)

Table 1

Short circuit current, $I_{\mathrm{SC}}$, open circuit voltage, $V_{\mathrm{OC}}$, fill factor, $\mathrm{FF}$, and conversion efficiency of the solar cells under study.

\begin{tabular}{lllll}
\hline Solar cell & $V_{\mathrm{OC}}(\mathrm{V})$ & $I_{\mathrm{SC}}(\mathrm{mA})$ & FF & Efficiency $(\%)$ \\
\hline REF & 0.61 & 4.38 & 80.6 & 15 \\
PDS-1 & 0.57 & 2.97 & 80.2 & 9.5 \\
PDS-2 & 0.57 & 3.01 & 80.6 & 9.9 \\
\hline
\end{tabular}

Control samples of SRO layers were deposited on Si wafers and characterized by X-ray photoelectron spectroscopy (XPS). The details about XPS analysis and techniques could be found in Ref. [12]. Optical properties of these films were investigated with variable-angle ellipsometric spectroscopy and PL spectroscopy. The ellipsometric spectra were measured from 300 to $1700 \mathrm{~nm}$ at different angles and the dielectric function obtained by a standard least square regression analysis. PL spectra were measured at room temperature with an excitation wavelength of $532 \mathrm{~nm}$ and the spectra have been normalized to the spectral response of the detector. Photoluminescence excitation (PLE) spectra were measured with a Cary Eclipse Fluorescence spectrometer. The wavelength of incident light was scanned from 400 to $680 \mathrm{~nm}$ for the PLE measurement observing the emission at $800 \mathrm{~nm}$.

Efficiency measurements were performed using an ABET sun 2000 solar simulator class $\mathrm{AAB}$ following closely the procedure described in the international standard CEI IEC 60904-1. In the following we indicate only the most important parts of the procedure. The irradiance measurements were performed using a PV reference device. The temperature of both solar cells and reference cells is actively controlled to be $24 \pm 1{ }^{\circ} \mathrm{C}$ during the measurements. $I-V$ curves were measured with a Semiconductor Source Measure Unit from Keithley Instruments Inc. using a 4 wire connection and the measurements are corrected for the external series resistance. Stability in time is continuously monitored by routinely repeating the measurements on c-Si solar cells. The maximum variation noticed in the time of observation (3 years) is less than 3\%. For other measurements, we follow the procedure outlined in 6.1 to 6.7 of IEC 60904-1. Short circuit current, ISc, open circuit voltage, $V_{\mathrm{OC}}$, and fill factor, $\mathrm{FF}$, given in Table 1 , were extracted from the $I-V$ curves taken under the conditions described above.

\section{Results and discussion}

After the high temperature annealing, Si-NCs precipitate inside the SRO layer [13]. This is confirmed by PL measurements (Fig. 2(a)), where emission bands peaked at 800 and $790 \mathrm{~nm}$ for PDS-2 and PDS-1, respectively. Due to the larger thickness, PDS-2 shows a stronger emission intensity than PDS-1. The difference is due to the larger optical density of PDS-2 than that of PDS-1, which means higher absorption as well as stronger emission. The PLE spectra of PDS-2 and PDS-1 devices are reported in Fig. 2(b). Si-NC light emission increases exponentially as the excitation photon wavelength decreases.

The reflectance spectra of the various cells were measured by ellipsometry. We used a thin film modeling software $\left(\right.$ Scout $\left.{ }^{\mathbb{R}}\right)$, and the optical parameters measured by an ellipsometer, to fit the reflectance spectra. From the simulation and by using the definition of Fig. 1 (bottom panel), we deduced the reflectance $\left(R_{\mathrm{SRO}}, R_{\mathrm{REF}}\right)$, the absorbance $\left(A_{\mathrm{SRO}}, A_{\mathrm{REF}}\right)$, and the transmittance $\left(T_{\mathrm{SRO}}, T_{\mathrm{REF}}\right)$ of the

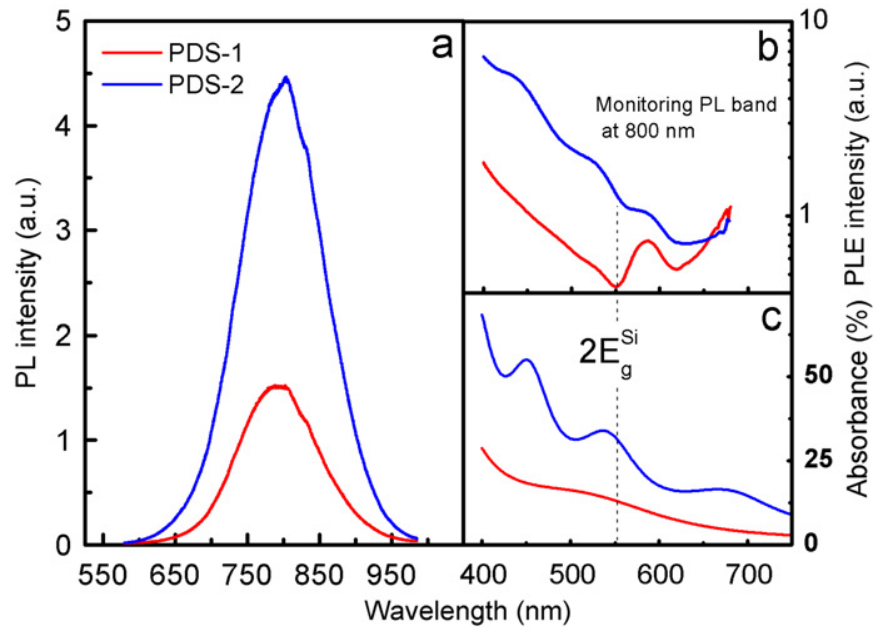

Fig. 2. (a) Photoluminescence (PL), (b) photoluminescence excitation (PLE), and (c) absorbance spectra of the two PDS devices. PL spectra were measured with an excitation wavelength of $532 \mathrm{~nm}$. Photoluminescence excitation spectra were measured by a Cary Eclipse Fluorescence set-up and the wavelength of incident light was scanned from 400 to $680 \mathrm{~nm}$.

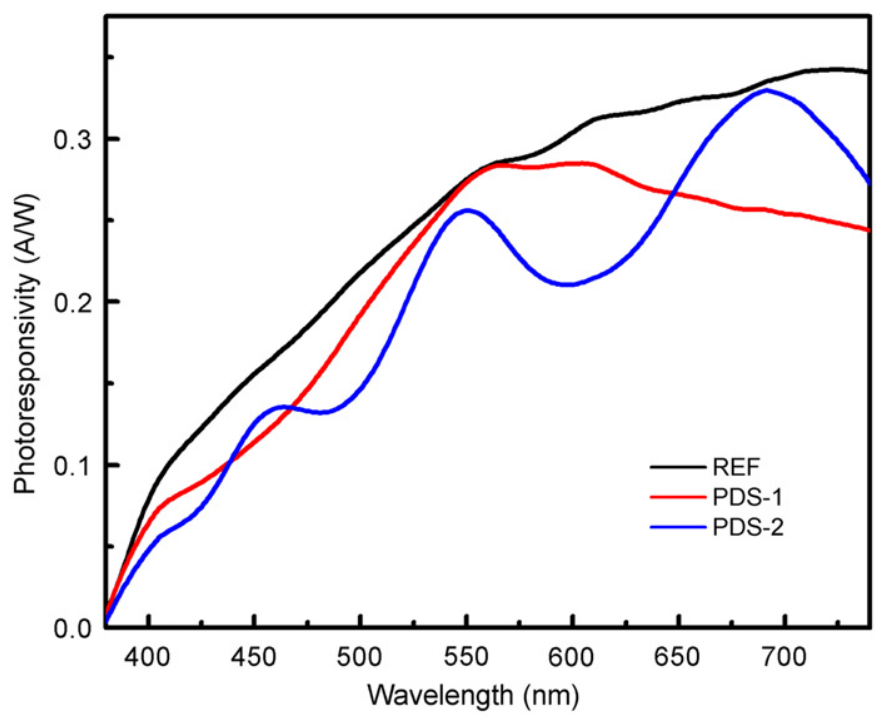

Fig. 3. Photoresponsivities (PR) of the devices under study. The PR were measured by illuminating the cell with a monochromatized $1000 \mathrm{~W}$ Xe lamp and measuring the resulting photocurrent. 
SRO and $\mathrm{SiO}_{2}$ layers for PDS-1, PDS-2, and REF cells. Note that $T_{\mathrm{SRO}}$ and $T_{\text {REF }}$ represent the fraction of the incident optical power, which actually reaches the active layer of the cells.

The absorbance spectra $A_{\mathrm{SRO}}$ are shown in Fig. 2(c). Interference patterns are observed due to internal reflections at the SRO interfaces (SRO/air and SRO/silicon). We can see that the absorbance of the thick SRO layer is larger than that of the thin SRO layer. By comparing the PL and absorbance spectra, we note that the SRO layers absorb mostly high energy photons and emit low energy photons due to the characteristic energy shift of Si-NCs between absorption and emission. This is important for down-shifting application since it demonstrates the lack of re-absorption in Si-NCs.

Fig. 3 reports the wavelength dependence of the photoresponsivity (PR) of the three solar cells studied: $\mathrm{PR}(\lambda)=i_{\mathrm{ph}}(\lambda) / P_{\mathrm{o}}(\lambda)$, where $i_{\mathrm{ph}}$ is the short circuit current and $P_{\mathrm{o}}$ is the incident optical power. The REF cell has the largest photoresponse over the whole investigated incident light wavelength range, due to the lower reflectance of the $\mathrm{SiO}_{2}$ layer than that of the SRO layers. On the other hand, PDS-1 and PDS-2 have PR spectra, which represent their optical transmission properties.

In order to verify the role of the SRO layers as down-shifter layer, we have extracted the down-shifting contribution of the Si-NC from their pure optical role, i.e. the role as antireflection coating. To this aim we

a

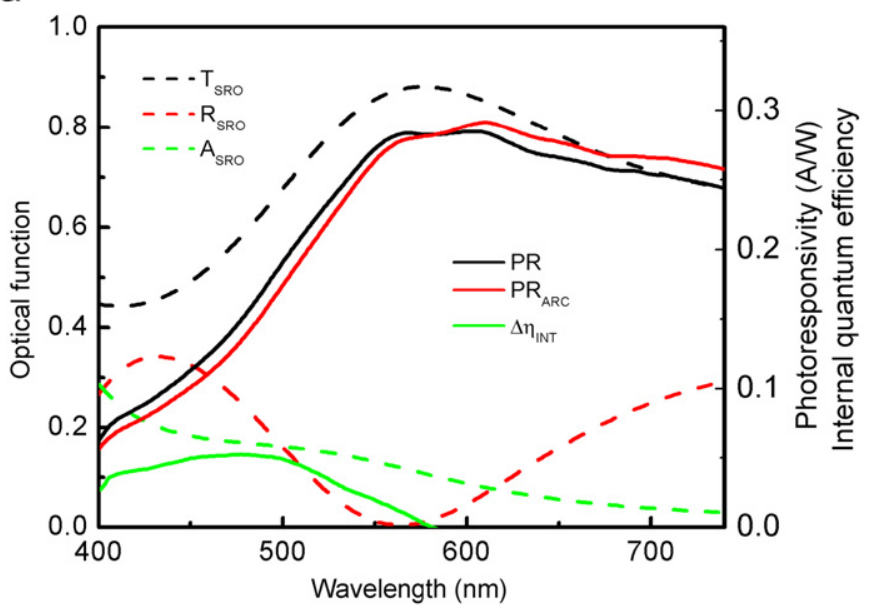

b

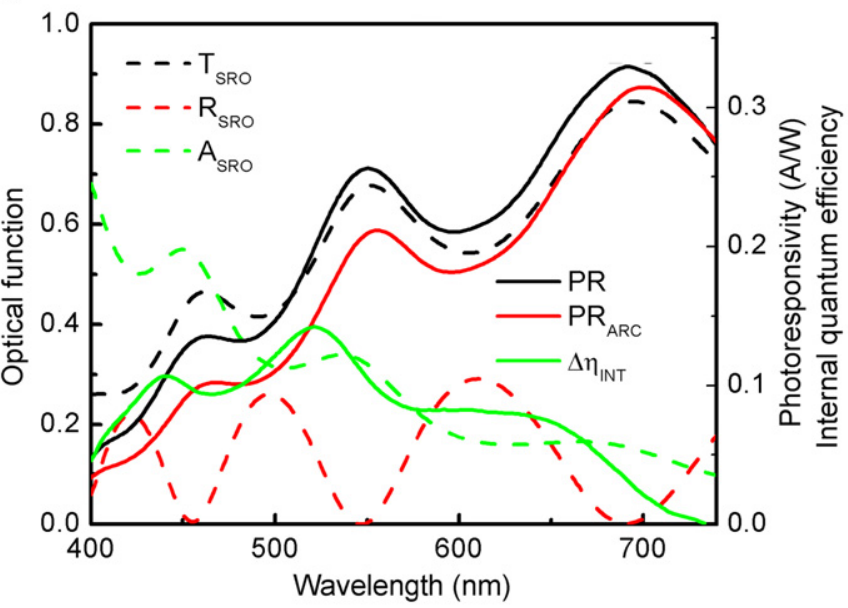

Fig. 4. Optical function (transmittance, black dashed line, reflectance, red dashed line, absorbance, green dashed line), measured photoresponsivity (PR, black line), modeled photoresponsivity without emission from the SRO layer ( $\mathrm{PR}_{\mathrm{ARC}}$, red line), and increment in the IQE ( $\Delta \eta_{\mathrm{INT}}$, green line) for PDS-1 (a) and PDS-2 (b) devices. (For interpretation of the references to color in this figure legend, the reader is referred to the web version of this article.) have proceeded in the following way. Let us call PR the measured photoresponsivity of the PDS cell and $\mathrm{PR}_{\mathrm{REF}}$ the measured photoresponsivity of the REF cell. Then the internal photoresponsivity of the silicon cell is given by $\mathrm{PR}_{\mathrm{INT}}=\mathrm{PR}_{\mathrm{REF}} / T_{\mathrm{REF}}$, which represents the photoresponse of silicon. In fact, $i_{\mathrm{ph}}=\mathrm{PR}_{\mathrm{INT}} \times P_{\mathrm{o}} \times T_{\mathrm{REF}}$ is the photocurrent produced in silicon due to the photons, which reach silicon, i.e. photons transmitted by the $\mathrm{SiO}_{2}$ layer. Since the underlying silicon is the same for the REF as well as for the PDS cells, we compute the expected PR of the PDS cells when the SRO layer acts as a bare antireflection coating as $\mathrm{PR}_{\mathrm{ARC}}=\left(\mathrm{PR}_{\mathrm{INT}}\right) \times T_{\mathrm{SRO}}$. Note that $\mathrm{PR}_{\mathrm{ARC}}$ is the expected result when the SRO is not playing any role as down-shifter, i.e. in absence of SRO luminescence. On the contrary, if the SRO layer absorbs high energy photons and then re-emits low energy photons, one expects a larger photoresponse due to the SRO emission, i.e. the measured $\mathrm{PR} \neq \mathrm{PR}_{\mathrm{ARC}}$. In particular, a measure of the difference in the internal quantum efficiency $\left(\Delta \eta_{\mathrm{INT}}\right)$ of the silicon cell due to the presence of the downshifter layer is $\Delta \eta_{\mathrm{INT}}(\lambda)=\left(\operatorname{PR}(\lambda)-\operatorname{PR}_{\mathrm{ARC}}(\lambda)\right)(1.24 / \lambda)\left(1 /\left(1-R_{\mathrm{SRO}}\right)\right)$, where $\lambda$ is in $\mu \mathrm{m}$.

The results of this modeling are reported in Fig. 4(a) for the PDS-1 cell and in Fig. 4(b) for the PDS-2 cell. PR and PR ARC $_{\text {differ }}$ mainly in the low wavelength region, where SRO has a larger absorbance. $\Delta \eta_{\text {INT }}$ is significantly larger for PDS-2 than for PDS-1. It peaks at a wavelength of about $500 \mathrm{~nm}$. With respect to the REF cell we got an enhancement of the IQE integrated on the measurements range of $380-740 \mathrm{~nm}$ of $0.8 \%$ for PDS- 1 and of $14 \%$ for PDS- 2 . The large enhancement for PDS-2 is due to both a stronger absorption and emission from the thicker SRO layer.

By using a solar simulator with the AM1.5G spectral filter we measured the conversion efficiency of our cells and found the value of $15 \%, 9.5 \%$, and $9.9 \%$ for REF, PDS-1, and PDS-2, respectively (Table 1). The decreased conversion efficiency of the PDS devices is mainly caused by the increased reflectance of the layer due to the large refractive index of the $\mathrm{SRO}$ with respect to the $\mathrm{SiO}_{2}$. Indeed, integrated reflectance in a range of $350-1200 \mathrm{~nm}$ is $10 \%, 21 \%$, and $16 \%$ for REF, PDS-1, and PDS-2, respectively.

\section{Conclusions}

In conclusion, PL down-shifting effects have been observed in silicon solar cell passivated by a Si-NC down-shifting layer. Through a careful analysis of the PR data, the role of the Si-NC as down-shifters has been demonstrated. In the visible photon region, a maximum enhancement of the IQE of $14 \%$ has been found. A proper design of the downshifter thickness could reduce the reflectivity of the devices, resulting in an overall conversion efficiency enhancement.

\section{Acknowledgements}

The authors would like to thank the support from Optoi Company and FP7 ICT-248909 project LIMA.

\section{References}

[1] M.A. Green, Third generation photovoltaics: solar cells for 2020 and beyond Physica E 14 (2002) 65-70.

[2] M.A. Green, Third generation photovoltaics: ultra-high conversion efficiency at low cost, Prog. Photovoltaics 9 (2001) 123-135.

[3] M.A. Green, Third Generation Photovoltaics: Advanced Solar Energy Conversion, Springer, Berlin, Germany, 2003.

[4] M.C. Beard, R.J. Ellingson, Multiple exciton generation in semiconductor nanocrystals: toward efficient solar energy conversion, Laser Photonics Rev. 2 (2008) 377-399.

[5] M.A. Green, Potential for low dimensional structures in photovoltaics, Mater Sci. Eng. B 74 (2000) 118-124.

[6] A.J. Nozik, Quantum dot solar cells, Physica E 14 (2002) 115-120.

[7] V.V. Kislyuk, O.P. Dimitriev, Nanorods and nanotubes for solar cells, J. Nanosci. Nanotechnol. 8 (2008) 131-148. 
[8] L. Tsakalakos, Nanostructures for photovoltaics, Mater. Sci. Eng. R 62 (2008) 175-189.

[9] C. Strumpel, M. McCann, G. Beaucarne, V. Arkhipov, A. Slaoui, V. Svrcek, C. del Canizo, I. Tobias, Modifying the solar spectrum to enhance silicon solar cell efficiency - an overview of available materials, Sol. Energy Mater. Sol. Cells 91 (2007) 238-249.

[10] W. van Sark, A. Meijerink, R. Schropp, J. van Roosmalen, E. Lysen, Enhancing solar cell efficiency by using spectral converters, Sol. Energy Mater. Sol. Cells 87 (2005) 395-409.
[11] V. Svrcek, A. Slaoui, J. Muller, Silicon nanocrystals as light converter for solar cells, Thin Solid Films 451-452 (2004) 384-388.

[12] S. Prezioso, A. Anopchenko, Z. Gaburro, L. Pavesi, G. Pucker, L. Vanzetti, P. Bellutti, Electrical conduction and electroluminescence in naocrystalline silicon-based light emitting devices, J. Appl. Phys. 104 (2008) 063103/1063103/8.

[13] L. Pavesi, R. Turan (Eds.), Silicon Nanocrystals: Fundamentals, Synthesis and Applications, Wiley-VCH Verlag GmbH, Berlin, 2010. 